Table 1 PTU-007 Relationship of Dukes staging at surgical resection to status within the Bowel Cancer Screening Programme

\begin{tabular}{lllll}
\hline Dukes staging at surgery & A & B & C1 & C2 \\
\hline FOBt positive $(n=20)$ & 8 & 4 & 7 & 1 \\
FOBt negative $(n=3)$ & 0 & 0 & 2 & 1 \\
Invited post diagnosis $(n=26)$ & 5 & 16 & 5 & 0 \\
No response to invitation $(n=13)$ & 4 & 5 & 4 & 0 \\
Not yet invited $(n=10)$ & 1 & 7 & 1 & 1 \\
Total $(n=72)$ & 18 & 32 & 19 & 3 \\
\hline
\end{tabular}

Results Two hundred and sixty five patients had resections for cancer and the age at time of surgery ranged from 34.5 to 91.7 years. There were 146 male and 119 female patients. Of these 24 patients were referred through the BCSP following a positive FOBt (4 aged over 70 opt-ins), 3 patients had completed a FOBt with a negative result. 72 patients were in the age range covered by the BCSP and the Dukes staging in relation to their status within the programme is given in table 1 which shows a significantly increased number of Dukes A tumours in patients aged 60-70 with a positive FOBt in the BCSP (Chi squared test $\mathrm{p}<0.05)$.

Eleven of the 20 FOBt positive patients aged 60-69 were symptomatic at the time of assessment within the programme. Three of the four over 70 opt-ins had symptoms with two Dukes B and two C1 tumours in this group

Conclusion BCSP detected cancers are at a significantly earlier stage than those detected outside the programme. The three patients with a negative FOBt in the programme had more advanced tumours.

Competing interests None.

Keywords bowel cancer, bowel cancer screening, bowel cancer staging.

\title{
PTU-007 IMPACT OF BOWEL CANCER SCREENING ON STAGING OF BOWEL CANCER
}

doi:10.1136/gut.2011.239301.135

B J Johnston, ${ }^{1 *}$ M Ali ${ }^{2}{ }^{1}$ Lady Sobell Gastrointestinal Unit, Wexham Park Hospital, Slough, UK; ${ }^{2}$ Department of Histopathology, Wexham Park Hospital, Slough, UK

Introduction The national Bowel Cancer Screening Programme (BCSP) aims to reduce mortality from bowel cancer by $16 \%$. Screening started in Berkshire in May 2008. To achieve this it is hoped that screen detected cancers will be diagnosed at an earlier stage than those detected in the routine service.

Methods A retrospective audit was carried out on all resection specimens received at one of the screening hospitals since the start of the BCSP. Cases were identified using the histopathology reporting system. Staging and tumour site information was gathered from the histopathology reports, referral route information from the cancer recording database and status in the BCSP from the bowel cancer screening computer system. Symptom profile for those patients with a positive FOBt was collected from the datasets collected at the colonoscopy assessment visit. 